\title{
Markov Chain Monte Carlo Solution of Poisson's Equation in Axisymmetric Regions
}

\author{
Adebowale E. Shadare ${ }^{1}$, Matthew N. O. Sadiku² and Sarhan M. Musa ${ }^{3}$ \\ ${ }^{1,2}$ Department of Electrical/Computer Engineering, Prairie View A\&M University, Texas, United States \\ ${ }^{3}$ Department of Engineering Technology, Prairie View A\&M University, Texas, United States \\ *Corresponding Author, Email: shadareadebowale@yahoo.com
}

\begin{abstract}
The advent of the Monte Carlo methods to the field of EM have seen floating random walk, fixed random walk and Exodus methods deployed to solve Poisson's equation in rectangular coordinate and axisymmetric solution regions. However, when considering large EM domains, classical Monte Carlo methods could be time-consuming because they calculate potential one point at a time. Thus, Markov Chain Monte Carlo (MCMC) is generally preferred to other Monte Carlo methods when considering whole-field computation. In this paper, MCMC has been applied to solve Poisson's equation in homogeneous and inhomogeneous axisymmetric regions. The MCMC results are compared with the analytical and finite difference solutions.
\end{abstract}

Index Terms - Poisson's equations, axisymmetric problem, inhomogeneous media, homogeneous medium, Markov Chain Monte Carlo (MCMC)

\section{Introduction}

Poisson's equation is an elliptic partial differential equation that frequently appears in many scientific problems such as electrostatics, surface reconstruction, gravitational problems, and semiconductors [1]-[2]. Due to its convenience, Poisson's equation in rectangular coordinate has been extensively studied using different numerical methods [3]-[5].

Stochastic methods such as the Monte Carlo techniques are nondeterministic numerical methods unlike the deterministic numerical methods such as finite difference, finite elements and moment methods used in solving mathematical and physical problems [6]. So Monte Carlo methods such as Floating random walk, Fixed random walk and Exodus methods have been used for solution of Poisson's equation in rectangular and axisymmetric regions [7]-[12]. With advancement in memory technology, MCMC method can handle EM problems in large solution domains within shortest possible time while serving as a viable alternative to other numerical methods.

In this paper, the solutions of axisymmetric Poisson's equations with the Markov Chain Monte Carlo are presented. Cases of homogeneous and inhomogeneous axisymmetric Poisson's equations are discussed. Simulation results are reported and they are compared with the finite difference method and analytical solutions. The solutions are found to be in close agreement.

\section{Poisson's Equation}

The Poisson's equation in solution region $R$ subject to Dirichlet boundary condition is given as

$$
\nabla^{2} V=-g(\rho, z)=-\frac{\rho_{S}}{\varepsilon}
$$

where $\rho_{S}$ is the surface charge; $\varepsilon=$ absolute permittivity and $V=V_{p}$ on Boundary $B$.

Assuming a square grid, the step size is given as $\Delta \rho=\Delta \boldsymbol{z}=\Delta$. Thus the finite difference equivalent of equation (1) is given as [13]

$$
\begin{aligned}
& V(\rho, z)=p_{\rho^{+}} V(\rho+\Delta, z)+p_{\rho-} V(\rho-\Delta, z) \\
& +p_{z+} V(\rho, z+\Delta)+p_{z^{-}} V(\rho, z-\Delta)+\frac{\Delta^{2} g}{4}
\end{aligned}
$$

If $\rho=i \Delta$ and $z=j \Delta$, the transition probabilities, $p_{z+}$, $p_{z-}, p_{\rho+}$ and $p_{\rho-}$, for axisymmetric homogeneous domains are given as [14]

$p_{z+}=p_{z-}=\frac{1}{4}, p_{\rho+}=\frac{1}{4}+\frac{1}{8 i}, p_{\rho-}=\frac{1}{4}-\frac{1}{8 i}$

The term $\frac{\Delta^{2} g}{4}$ is the source term that must be recorded at each step of the random walk.

The system is stochastic, thus

$p_{z+}+p_{z-}+p_{\rho+}+p_{\rho-}=1$

At $\rho=0$, the finite difference equivalence of equation (2) becomes [14]

$$
V(0, z)=p_{\rho+} V(\Delta, z)+p_{z+} V(0, z+\Delta)+p_{z-} V(0, z-\Delta)
$$

where $p_{\rho+}=\frac{4}{6}, p_{\rho-}=0, p_{z+}=p_{z-}=\frac{1}{6}$ 


\section{Markov Chain Monte Carlo}

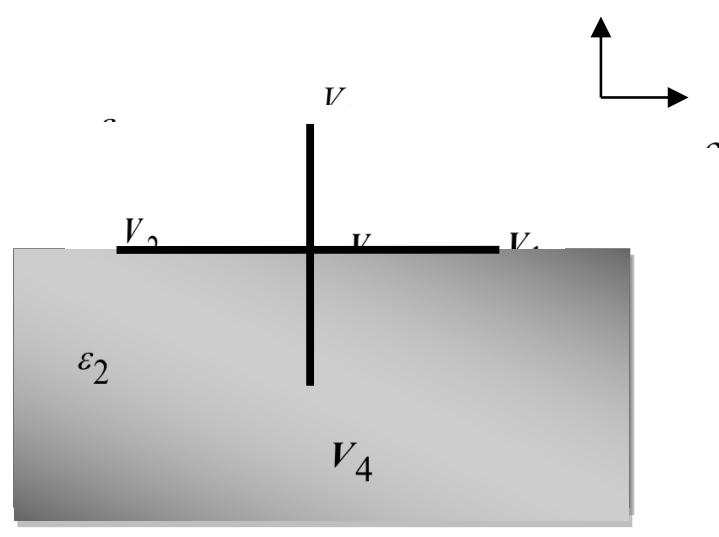

Figure 1. Interface between media of dielectric permittivity

$$
\varepsilon_{1} \text { and } \varepsilon_{2}[7] \text {. }
$$

\section{Inhomogeneous Media}

Inhomogeneous media occur when two or more media have variations in dielectric permittivity. The Figure 1 shows transition probabilities at a node at the interface between two dielectric permittivity $\varepsilon_{1}$ and $\varepsilon_{2}$.

For the $z=$ constant interface, $\varepsilon_{1} \frac{\partial V_{1}}{\partial z}=\varepsilon_{2} \frac{\partial V_{2}}{\partial z}$, and the transition probabilities at the interface are determined as [14]:

$$
\begin{aligned}
& p_{z+}=\frac{\varepsilon_{1}}{2\left(\varepsilon_{1}+\varepsilon_{2}\right)} \\
& p_{z-}=\frac{\varepsilon_{2}}{2\left(\varepsilon_{1}+\varepsilon_{2}\right)} \\
& p_{\rho+}=\frac{1}{4}+\frac{1}{8 i} ; p_{\rho-}=\frac{1}{4}-\frac{1}{8 i}
\end{aligned}
$$

Similarly, for $\rho=$ constant interface, $\frac{\varepsilon_{1}}{\rho} \frac{\partial V_{1}}{\partial \rho}=\frac{\varepsilon_{2}}{\rho} \frac{\partial V_{2}}{\partial \rho}$, and the transition probabilities are given as [15]:

$$
\begin{aligned}
& p_{\rho+}=\frac{\varepsilon_{1}}{2\left(\varepsilon_{1}+\varepsilon_{2}\right)}\left(1+\frac{\Delta}{2 \rho}\right) \\
& p_{\rho-}=\frac{\varepsilon_{2}}{2\left(\varepsilon_{1}+\varepsilon_{2}\right)}\left(1-\frac{\Delta}{2 \rho}\right) \\
& p_{z+}=p_{z-}=\frac{1}{4} .
\end{aligned}
$$

In this paper, $z=$ constant interface is used for the inhomogeneous problem discussed in the section $\mathrm{V}$.
Given a sequence of random variables $X^{(0)}, X^{(1)}, \ldots, X^{(n)}$ with probability distribution of $X^{(n)}$ determined by the probability distribution of $X^{(n-1)}$ [16]-[22]. The sequence is referred to as a Markov Chain with time-invariant conditional probability distributions. Markov chains are mathematical models that remember only the most recent past. In this paper, the Markov chain is the random walk while the discrete states are the grid nodes. The transition probability $P_{i j}$ is defined as the probability that a randomly walking particle at node $i$ will move to node $j$ and it is expressed as,

$$
\begin{aligned}
& P_{i j}=P\left(x_{n+1}=j \mid x_{0}, x_{1}, \ldots, x_{n}\right)= \\
& P\left(x_{n+1}=j \mid x_{n}\right), \quad j \in X, n=0,1,2, \ldots
\end{aligned}
$$

The transition probabilities are derived from the finite difference equivalence equation and they correspond to the random walks that form the background to the MCMC method.

The transition probability $\mathbf{P}$ is defined as

$$
\mathrm{P}=\left[\begin{array}{cccc}
\mathrm{P}_{00} & \mathrm{P}_{01} & \mathrm{P}_{02} & \ldots \\
\mathrm{P}_{10} & \mathrm{P}_{11} & \mathrm{P}_{12} & \ldots \\
\mathrm{P}_{20} & \mathrm{P}_{21} & \mathrm{P}_{22} & \ldots \\
\ldots & \ldots & \ldots & \ldots
\end{array}\right]
$$

$\mathbf{P}$ is stochastic and it is given by,

$$
\sum_{j \in X} P_{i j}=1, \quad i \in X
$$

If $n_{f}$ are free (non-absorbing) nodes and $n_{p}$ are fixed (absorbing) nodes, the size of $\mathbf{P}$ is $n \times n$ given as

$$
n=n_{f}+n_{p}
$$

Also, $n_{p}$ are numbered first and $n_{f}$ are numbered last, then $n \times n$ transition matrix, $\mathbf{P}$ becomes

$$
\mathbf{P}=\left[\begin{array}{ll}
\mathbf{I} & \mathbf{0} \\
\mathbf{R} & \mathbf{Q}
\end{array}\right]
$$

where

$n_{f} \times n_{p} \mathbf{R}$ matrix is the probabilities of moving from nonabsorbing nodes to absorbing ones;

$n_{f} \times n_{f} \mathbf{Q}$ matrix is the probabilities of moving from one non-absorbing node to another; 
$n_{f} \times n_{f} \quad I$ matrix is the identity matrix representing transitions between the absorbing nodes $\left(P_{i i}=1\right.$ and $\left.P_{i j}=0\right)$.

$n_{p} \times n_{f} \mathbf{0}$ matrix is the null matrix indicating no transitions from absorbing to non-absorbing nodes.

From equation 3, the elements of matrix $\mathbf{Q}$ at nodes in the $\rho \neq 0$ region are obtained summarily as

$Q_{i j}=\left\{\begin{array}{l}\frac{1}{4}, \quad \text { if } i \text { is directly connected to } j \\ \left(\frac{2 i+1}{8 i}\right), \quad \text { if } i \text { is directly connected to } i+1 \\ \left(\frac{2 i-1}{8 i}\right), \quad \text { if } i \text { is directly connected to } i-1 \\ 0, \quad \text { if } i=j \text { or } i \text { is not directly connected to } j\end{array}\right.$

Similarly, from equation 5, the elements of matrix $\mathbf{Q}$ for nodes at the line of Symmetry, $\rho=0$, are obtained as

$$
Q_{i j}= \begin{cases}\frac{1}{6}, & \text { if } i=0 \text { and is directly connected } \\ & \text { to } j \text { and } j-1 \\ \frac{4}{6}, & \text { if } i=0 \text { and is directly connected } \\ & \text { to } i+1 \\ 0, & \text { if } i=j \text { or } i \text { is not directly connected } \\ & \text { to } j\end{cases}
$$

The elements of matrix $R_{i j}$ are obtained in the same manner from equations (13) and (14) except that $j$ is an absorbing node.

The fundamental matrix, $\mathbf{N}$ for any absorbing Markov chain is given as,

$$
\mathbf{N}=(\mathbf{I}-\mathbf{Q})^{-1}
$$

where $N_{i j}$ is the average number of times that the randomly walking particle starting at node $i$ will pass through node $j$ before being absorbed.

The absorption probability matrix $\boldsymbol{B}$ is thus

$$
\mathbf{B}=\mathbf{N} \mathbf{R}
$$

where $n_{f} \times n_{p} B_{i j}$ matrix is the probability of moving from non-absorbing node $i$ to absorbing node $j$. The $\mathbf{B}$ matrix is stochastic and it is given as

$$
\sum_{j=1}^{n_{p}} B_{i j}=1, \quad i=1,2, \ldots, n_{f}
$$

Thus,

$$
\mathrm{V}_{f}=\mathrm{BV}_{p}+N G_{f}
$$

where $\mathbf{V}_{f}$ and $\mathbf{V}_{p}$ are the free and fixed nodes potentials respectively.

The term $G_{f}=\frac{\Delta^{2}}{4} g(\rho, z)$ is the vector of interior point contributions to be recorded at every step of the random walk [13]. The first term in RHS of equation (18), $\mathbf{B} \mathbf{V}_{\mathrm{p}}$ is used to evaluate the Laplace's equation. The second term, $\mathbf{N G}_{\mathrm{f}}$ in equation (18) is used for the analysis of Poisson's equation provided that $\mathbf{V}_{\boldsymbol{p}}=0$. Otherwise, the equation (18) is used for analysis of Poisson's equation for prescribed potential, $\mathbf{V}_{\boldsymbol{p}} \neq 0$.

In terms of the prescribed potentials $\boldsymbol{V}_{1}, \boldsymbol{V}_{2}, \ldots, \boldsymbol{V}_{\boldsymbol{n} \boldsymbol{p}}$, the first term in the RHS of equation (18), $\mathrm{V}_{\mathrm{f}}=\mathbf{B V}_{\mathrm{p}}$ becomes

$$
V_{i}=\sum_{j=1}^{n_{p}} B_{i j} V_{j}, i=1,2, \ldots, n_{f}
$$

where $V_{i}$ is the potential at any free node $i$. The Equation (19) provides solution at all the free nodes at once.

In this paper, Poisson's equation in homogeneous and inhomogeneous axisymmetric domains is presented where the boundary (prescribed) potentials are zeros, that is, $\mathbf{V}_{\boldsymbol{p}}=0$. So the equation (18) reduces to

$$
\mathbf{V}_{f}=N G_{f}
$$

where $\mathbf{V}_{f}$ are the free nodes potentials;

$N$ is the fundamental matrix;

$\mathrm{G}_{f}$ is the vector of the contributions of interior points. 


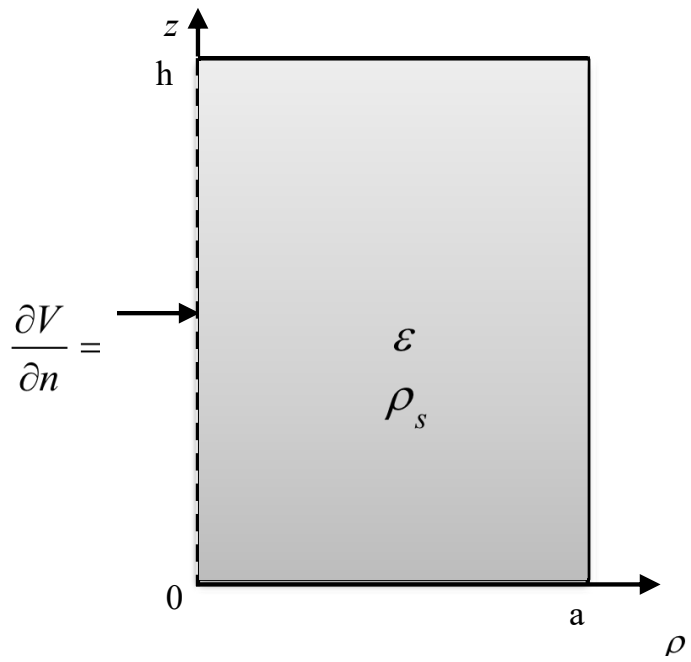

Figure 2. Axisymmetric Homogeneous Domain Approximation of a cylindrical tank of radius $a$ and height $h$.

\section{Simulation Results}

\section{A. Simulation Results for Axisymmetric Homogeneous Poisson's Equation}

Poisson's equation in axisymmetric homogeneous domain is given as:

$\frac{1}{\rho} \frac{\partial}{\partial \rho}\left(\rho \frac{\partial V}{\partial \rho}\right)+\frac{\partial^{2} V}{\partial z^{2}}=-\frac{\rho_{s}}{\varepsilon}$

Subject to boundary conditions:

$V(a, z)=0 ; V(a, h)=0 ; V(a, 0)=0$ and

$\frac{\partial V}{\partial n}=0$ at $\rho=0$

Suppose the axisymmetric domain of an earthed metal cylindrical tank of radius $\boldsymbol{a}$ and height $\boldsymbol{h}$ shown in the Figure 2 is completely filled with a charged liquid such as hydrocarbon. The Neumann boundary condition is imposed at the line of symmetry while the Dirichlet boundary conditions are imposed on the remaining three boundaries with zero potential. The analytical solution to the problem is presented in [8] as:

$$
V(\rho, z)=\frac{2 \rho_{s}}{\varepsilon_{0} \varepsilon_{r} a} \sum_{n=1}^{\infty} \frac{J_{0}\left(\lambda_{n} \rho\right)}{\lambda^{3} J_{1}\left(\lambda_{n} a\right)}\left(\begin{array}{l}
1-\cosh \left(\lambda_{n} z\right) \\
+\frac{\sinh \left(\lambda_{n} z\right)}{\sinh \left(\lambda_{n} h\right)}\left[\cosh \left(\lambda_{n} h\right)-1\right]
\end{array}\right)
$$

Table 1. Parameters for Poisson's Equation in Axisymmetric Homogeneous Domain.

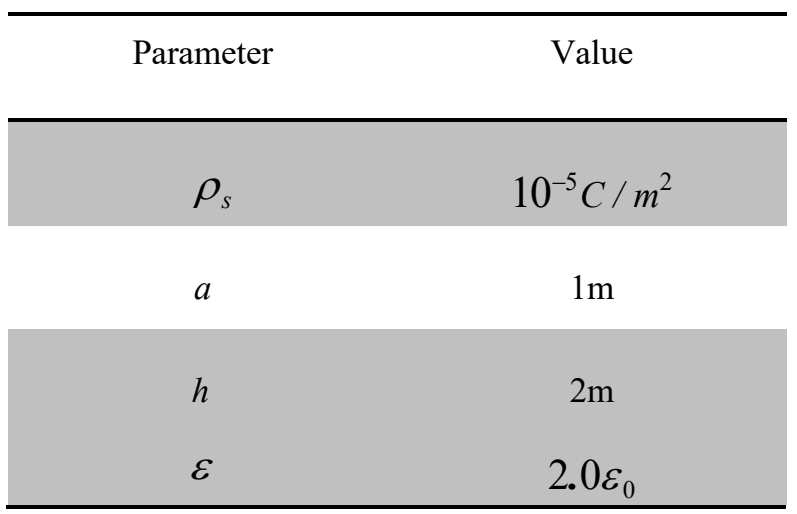

where $\lambda_{n}$ are the roots of $J_{0}\left(\lambda_{n} a\right)=0$. $J_{0}$ and $J_{1}$ are Bessel functions of first kind, order zero and one respectively.

With the background theory of the MCMC method discussed in the preceding section, all the simulations in this paper are carried out. Using the parameters in the Table 1, the results for the potential distributions along $\rho=0.5 m, 0 \leq z \leq 2 m, \rho=0,0 \leq z \leq 2 m$ (line of symmetry) and that along $\rho=0.9 m, 0 \leq z \leq 2 \mathrm{~m}$, are reported in the Figures 3(a)-(c). Similarly, the potential distribution along $z=1,0 \leq \rho \leq 1 \mathrm{~m}$, the surface plot and contour plot for all the grid nodes are presented in the Figure 3(d)-(f).

In the Table 2, the MCMC results for some randomly selected grid points are compared with the finite difference solution and analytical solution. The MCMC solution is, as evident, more accurate than the finite difference solution even after the step size is reduced from $0.05 \mathrm{~m}$ to $0.025 \mathrm{~m}$ as shown in the Table 2.

However, the number of iterations for the finite difference method increased from 500 to 5000 with the step size reduction. For problems with larger domain size, this could increase the computation time and memory requirement significantly. The solution to the same problem using the floating random walk Monte Carlo method with different domain size is reported in [8]. 
Table 2. Comparison for Analytical, FDM and MCMC for Poisson's Equation in Axisymmetric Homogeneous Domain.

\begin{tabular}{|c|c|c|c|c|c|}
\hline $\begin{array}{l}\text { Coordinate } \\
(\rho, z)\end{array}$ & $\begin{array}{l}\text { Analytical } \\
(\mathrm{KV})\end{array}$ & $\begin{array}{c}\text { FDM } \\
(\mathrm{KV}) \\
\Delta=0.05 \\
\text { Iteration }=500\end{array}$ & $\begin{array}{c}\text { FDM } \\
(\mathrm{KV}) \\
\Delta=0.025 \\
\text { Iteration }=5000\end{array}$ & $\begin{array}{c}\mathrm{MCMC} \\
(\mathrm{KV}) \\
\Delta=0.05\end{array}$ & $\begin{array}{c}\text { MCMC } \\
(\mathrm{KV}) \\
\Delta=0.025\end{array}$ \\
\hline$(0.25,0.5)$ & 86.870 & 86.042 & 86.784 & 86.486 & 86.849 \\
\hline$(0.5,0.5)$ & 71.730 & 71.212 & 71.715 & 71.409 & 71.743 \\
\hline$(0.75,0.5)$ & 44.080 & 43.881 & 44.130 & 43.942 & 44.141 \\
\hline$(0.5,0.75)$ & 83.662 & 83.019 & 83.643 & 83.030 & 83.677 \\
\hline$(0.5,0.25)$ & 46.838 & 46.543 & 46.834 & 46.701 & 46.851 \\
\hline
\end{tabular}

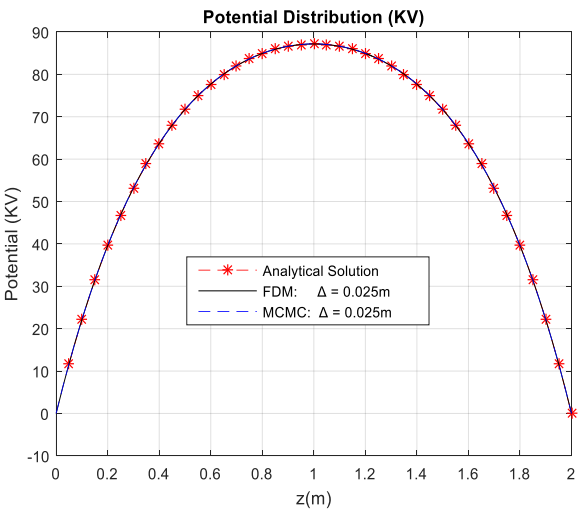

(a)

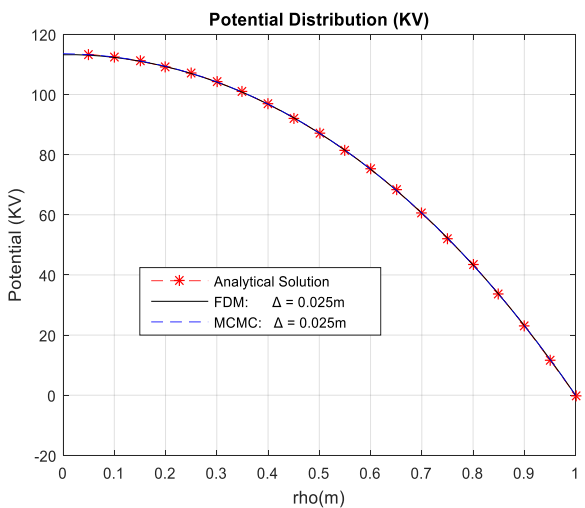

(d)

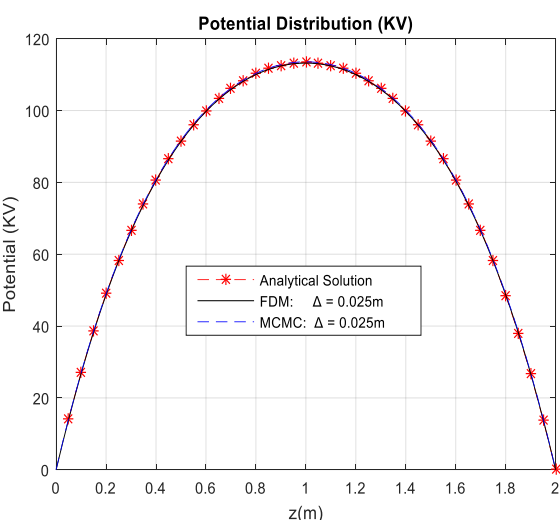

(b)

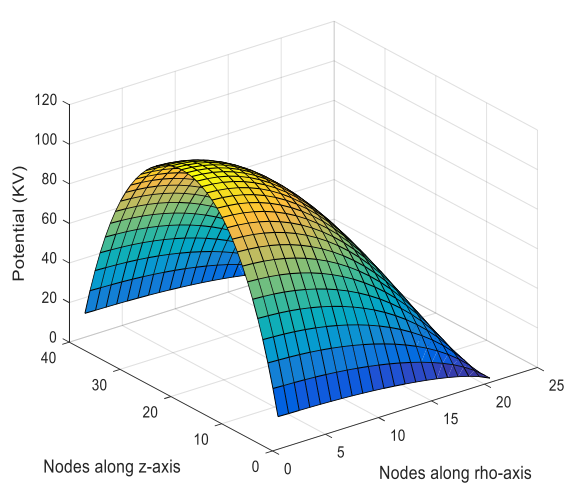

(e)

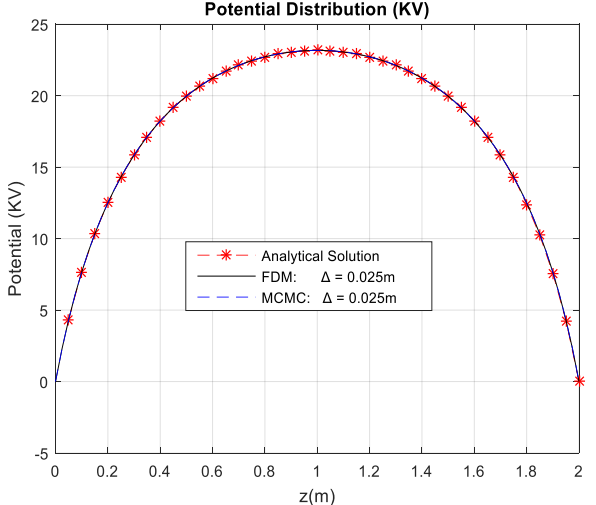

(c)

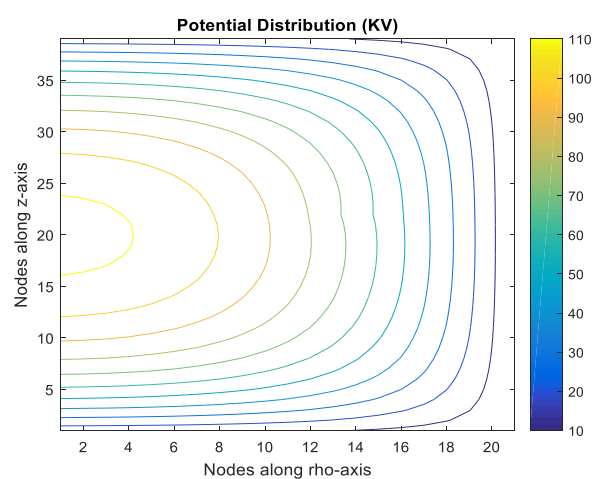

(f)

Figure 3. Potential Distribution along (a) $\rho=0.5 m, 0 \leq z \leq 2 m$ (b) Line of symmetry, $\rho=0,0 \leq z \leq 2 m$ (c) $\rho=0.9 m, 0 \leq z \leq 2 m$ (d) $z=1,0 \leq \rho \leq 1 m$ (e) Surface plot (f) Contour Plot for Poisson's equation in Homogeneous Axisymmetric Domain. 


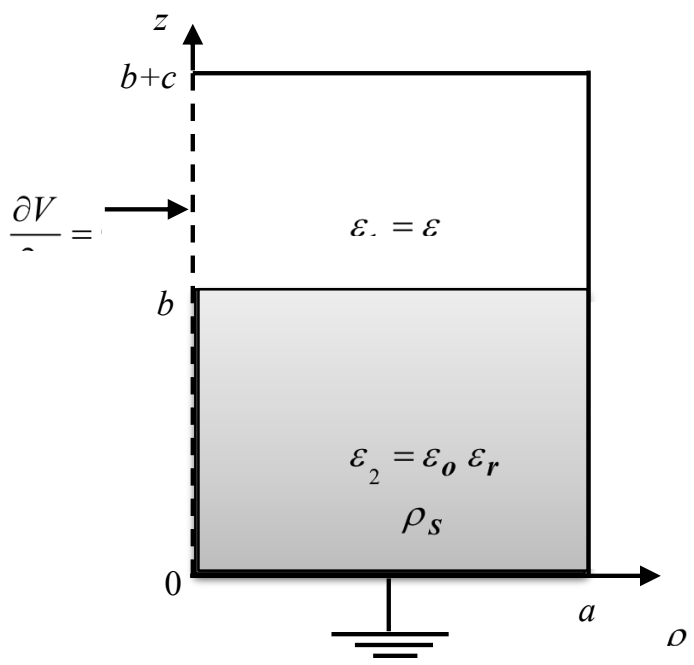

Figure 4. Inhomogeneous Axisymmetric Solution Region.

\section{B. Simulation Results for Axisymmetric Inhomogeneous Poisson's Equation}

Poisson's equation in axisymmetric inhomogeneous domain is given as [14], [23]-[24]:

$$
\begin{aligned}
& \frac{1}{\rho} \frac{\partial}{\partial \rho}\left(\rho \frac{\partial V_{l}}{\partial \rho}\right)+\frac{\partial^{2} V_{l}}{\partial z^{2}}=-\frac{\rho_{S}}{\varepsilon} \\
& \frac{1}{\rho} \frac{\partial}{\partial \rho}\left(\rho \frac{\partial V_{g}}{\partial \rho}\right)+\frac{\partial^{2} V_{g}}{\partial z^{2}}=0
\end{aligned}
$$

Subject to boundary conditions (B.C):

$$
\begin{aligned}
& V(a, z)=0 ; V_{g}(a, b+c)=0 ; V(a, 0)=0 ; V_{g}=V_{l}, z=b ; \\
& \frac{\partial V_{g}}{\partial z}=\varepsilon_{r} \frac{\partial V_{l}}{\partial z}, z=b \text { and } \frac{\partial V}{\partial n}=0 \text { at } \rho=0 .
\end{aligned}
$$

Suppose an earthed metal cylindrical tank is partly filled with a charged liquid such as hydrocarbon as shown in the Figure 4. The problem is described mathematically in equations (24) - (25). From the Figure 4, region $\boldsymbol{z}>\boldsymbol{b}$ is filled with gas, posing Laplace's equation problem while $\boldsymbol{z}<\boldsymbol{b}$ region is filled with charged hydrocarbon, constituting Poisson's equation. The analytical solution for the problem is presented in [14], [23]-[24]:

$$
V(\rho, z)=\left\{\begin{array}{c}
\sum_{n=1}^{\infty} \frac{2 \rho_{s}}{R_{n} K_{n}} J_{0}\left(\lambda_{n} \rho\right)\left[\cosh \left(\lambda_{n} b\right)-1\right] \sinh \left[\lambda_{n}(b+c-z)\right] \\
z \geq b \\
\sum_{n=1}^{\infty} \frac{2 \rho_{s}}{R_{n} \varepsilon_{r}} J_{0}\left(\lambda_{n} \rho\right)\left(\begin{array}{l}
\frac{\sinh \left(\lambda_{n} z\right)}{K_{n}}\left[\cosh \left(\lambda_{n} b\right) \cosh \left(\lambda_{n} c\right)\right. \\
+\varepsilon_{r} \sinh \left(\lambda_{n} b\right) \sinh \left(\lambda_{n} c\right) \\
\left.-\cosh \left(\lambda_{n} c\right)\right]-\cosh \left(\lambda_{n} z\right)+1 \\
z \leq b
\end{array}\right.
\end{array}\right.
$$

where $K_{n}=\sinh \left(\lambda_{n} b\right) \cosh \left(\lambda_{n} c\right)+\varepsilon_{r} \cosh \left(\lambda_{n} b\right) \sinh \left(\lambda_{n} c\right)$;

$$
R_{n}=\varepsilon_{0} a \lambda_{n}^{3} J_{1}\left(\lambda_{n} a\right) ; \lambda_{n} \text { are the roots of } J_{0}\left(\lambda_{n} a\right)=0 \text {. }
$$

$J_{0}$ and $J_{1}$ are Bessel functions of first kind, order zero and one.

With the parameters in the Table 3, the MCMC in this section is essentially the same as in the previous section except that the transition probabilities at the media interface are described by the equations (6). The potential distributions along $\rho=0.5 m, 0 \leq z \leq 2 m, \rho=0,0 \leq z \leq 2 m$ (line of symmetry) and $\rho=0.9 m, 0 \leq z \leq 2 m$, are reported in the Figures 5(a)-(c). Also, the potential distribution at the media interface, $z=1,0 \leq \rho \leq 1 \mathrm{~m}$, surface and contour plots are presented in the Figure 5(d)-(f). The solution to the same problem using finite difference method and Exodus method are presented in [7] and [14].

The MCMC solutions for selected grid points are compared with the FDM and analytical solution as in the Table 4. With $\Delta=0.05 \mathrm{~m}$, the MCMC solution is much more accurate than the FDM when compared with the analytical solution. Further reduction in $\Delta$ from $0.05 \mathrm{~m}$ to $0.005 \mathrm{~m}$ for the FDM with 100,000 iteration steps gives accurate solution that agrees with the MCMC and analytical solutions.

However, the computation time for the FDM increased from 0.0253 seconds to $7 \mathrm{mins}$ and 30 seconds compared to MCMC which is 0.06281 seconds. The MCMC agrees perfectly with the analytical solution while the FDM requires further reduction in step size to converge to the analytical solution.

Table 3. Parameters for Inhomogeneous Poisson's Problem.

\begin{tabular}{cc}
\hline Parameter & Value \\
\hline$\rho_{S}$ & $10^{-5} C / m^{2}$ \\
$a$ & $1 \mathrm{~m}$ \\
$b=c$ & $1 \mathrm{~m}$ \\
$\varepsilon_{1}$ & $\varepsilon_{0}$ \\
$\varepsilon_{2}$ & $2.0 \varepsilon_{0}$ \\
\hline
\end{tabular}


Table 4. Comparison for Analytical, FDM and MCMC for Axisymmetric Inhomogeneous Problem.

\begin{tabular}{|c|c|c|c|c|}
\hline $\begin{array}{l}\text { Coordinate } \\
\qquad(\rho, z)\end{array}$ & $\begin{array}{c}\text { Analytical } \\
(\mathrm{KV})\end{array}$ & $\begin{array}{c}\text { FDM } \\
(\mathrm{KV}) \\
\Delta=0.05 \mathrm{~m} \\
\text { Iteration }=500\end{array}$ & $\begin{array}{c}\text { FDM } \\
(\mathrm{KV}) \\
\Delta=0.005 \mathrm{~m} \\
\text { Iteration }=100,000\end{array}$ & $\begin{array}{c}\text { MCMC } \\
(\mathrm{KV}) \\
\Delta=0.05 m\end{array}$ \\
\hline$(0.25,0.3)$ & 57.8821 & 56.7096 & 57.8192 & 58.0019 \\
\hline$(0.35,1.5)$ & 19.3922 & 17.7028 & 19.2520 & 19.7284 \\
\hline$(0.5,1.05)$ & 51.4958 & 47.3810 & 51.1197 & 52.2268 \\
\hline$(0.6,1.6)$ & 9.6358 & 8.7791 & 9.5643 & 9.8021 \\
\hline$(0.8,0.6)$ & 34.8676 & 34.0749 & 34.8509 & 34.8935 \\
\hline
\end{tabular}

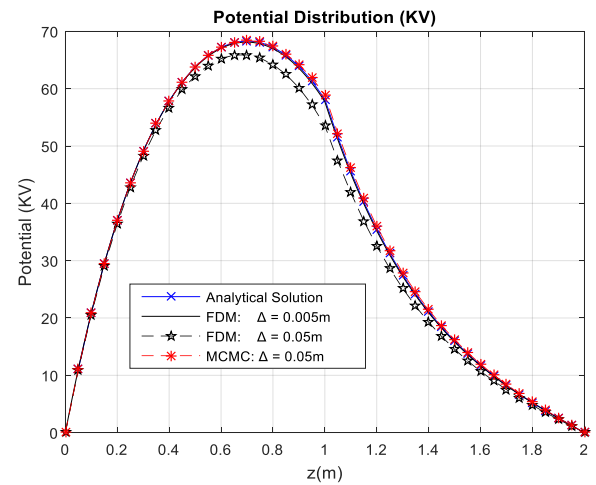

(a)

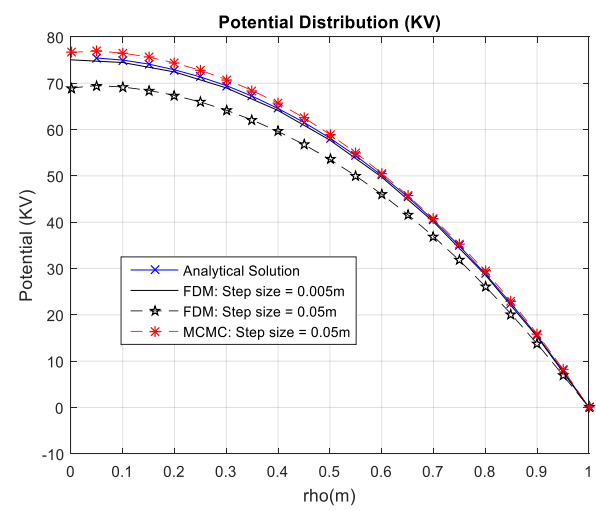

(d)

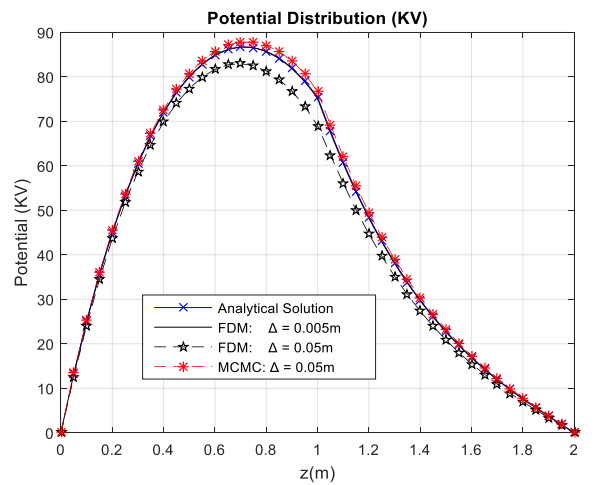

(b)

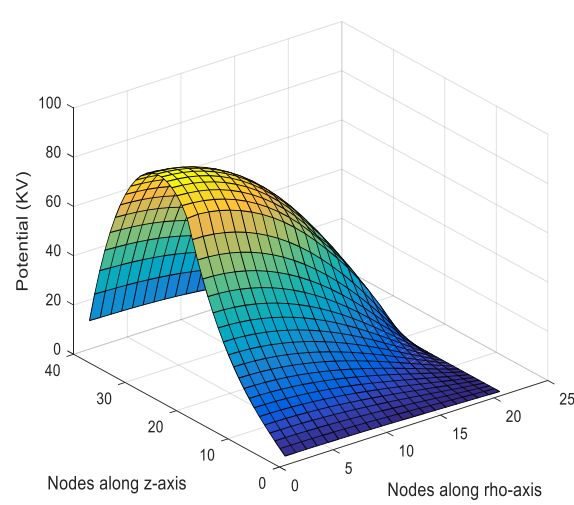

(e)

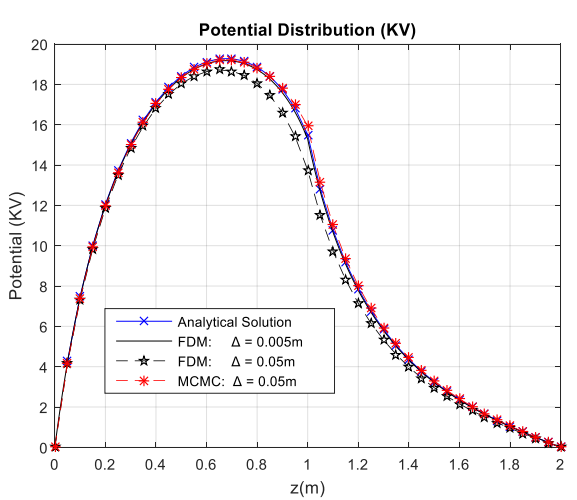

(c)

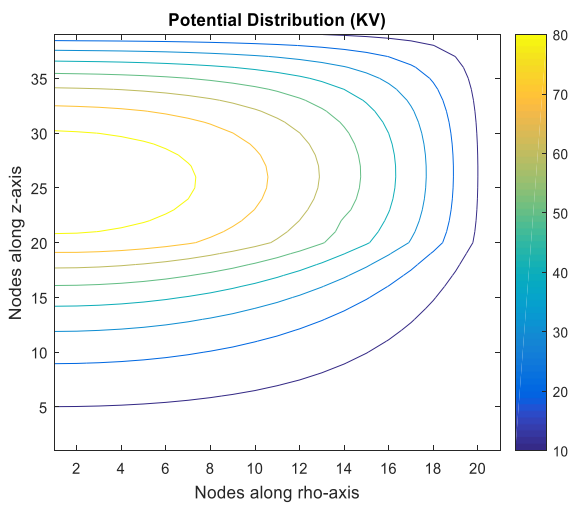

(f)

Figure 5. Potential Distribution along (a) $\rho=0.5 m, 0 \leq z \leq 2 m$ (b) Line of symmetry, $\rho=0,0 \leq z \leq 2 m$ (c) $\rho=0.9 m, 0 \leq z \leq 2 m$ (d) Media interface, $z=1,0 \leq \rho \leq 1 m$ (e) Surface plot (f) Contour Plot for Poisson's equation in Inhomogeneous Axisymmetric Domain. 


\section{Conclusion}

Poisson's equation has been extensively studied using different numerical methods and analytical method for few simple cases. Thus, for complex problems, there is a need for continuous development of simple and efficient numerical methods. Since the introduction of probabilistic method such as Monte Carlo method to the field of electromagnetics, several Monte Carlo methods developed such as floating random walk, fixed random walk and Exodus method have been used to solve Poisson's equation, notably in rectangular coordinate and axisymmetric solution regions. However, these methods calculate potentials one point at a time and are time-consuming when solving complex electromagnetic problems. In this paper, the MCMC method which is an improvement on the classical Monte Carlo method has been applied to solve Poisson's equations in axisymmetric region in homogeneous and inhomogeneous regions. The MCMC solutions reported in this paper are compared with analytical and finite difference method. In the case of homogeneous problem, the MCMC method agrees with the analytical and the finite difference method with difference in computation time being only a fraction of seconds. In the case of inhomogeneous problem, the MCMC method agrees perfectly with the analytical solution. However, further reduction in step size is required for the finite difference solution to converge to the analytical solution with attendant increase in iteration steps and computation time.

\section{References}

[1] A. Buonomo and C. Di Bello, “On Solving Poisson's Equation in Two-Dimensional Semiconductor Devices," Electronics Letters, February 1984, vol. 20, no. 4, pp. 156-158.

[2] https://en.wikipedia.org/wiki/Poisson $\% 27$ s_equation

[3] M. N. O. Sadiku et al., "A Further Introduction to Finite Element Analysis of Electromagnetic Problems," IEEE Transactions on Education, vol. 34, no. 4, November 1991, pp. 322-329.

[4] Chen CS, Muleshkov AS, Golberg MA, Mattheij RMM. "A mesh-free approach to solving the axisymmetric Poisson's equation," Numerical Methods Partial Differential Equation, 2005, vol. 21, 349-367.

[5] R. C. Garcia and M.N.O. Sadiku, "Neuro-Monte Carlo Solution of Electrostatic Problems," Journal of Franklin Institute, vol. 335B, no. 1, 1998, pp. 53-69.

[6] M. N. O. Sadiku, "Monte Carlo Methods in an Introductory Electromagnetic Course," IEEE Transactions on Education, vol. 33, no.1, February 1990, pp. 73-80.

[7] M. N. O. Sadiku, S. O. Ajose, and Zhibao Fu, "Applying the Exodus Method to Solve Poisson's Equation," IEEE Trans. Microwave Theory and Techniques, vol. 42, no.4, April, 1994, pp. 661-666.

[8] M. N. O. Sadiku and R. C. Garcia, "Monte Carlo Floating Random Walk Solution of Poisson's Equation," Proceedings of Southeastcon, 1993.
[9] J. M. Delaurentis and L. A. Romero, "A Monte Carlo Method for Poisson's Equation," Journal of Computational Physics, 1990, vol. 90, pp. 123-140.

[10] M. N. O. Sadiku and D. T. Hunt, "Solution of Dirichlet Problems by the Exodus Method," IEEE Transactions on Microwave Theory and Techniques, 40, 1, January 1992, pp. 89-95.

[11]R. C. Garcia and M. N. O. Sadiku, "Monte Carlo FixedRadius Floating Random Walk Solution for Potential Problems," Proceedings of the IEEE Southeastcon, 1996, pp. 88-91.

[12] M. N. O. Sadiku and K. Gu, "A New Monte Carlo Solution For Neumann Problems," Proc. IEEE Southeastcon, April, 1996, pp. 92-95.

[13] M. N. O. Sadiku, et al., "Markov Chain Monte Carlo Solution of Poisson's Equation," International Journal on Recent and Innovation Trends in Computing and Communication, vol. 3, Issue 1, 2015, pp. 106-112.

[14] M. N. O. Sakidu, Computational Electromagnetics with MATLAB. Boca Raton, FI, Boca Raton, CRC Press, Fourth Edition, 2019.

[15]M. N. O. Sadiku, "Monte Carlo Solution of Axisymmetric Potential Problems," IEEE Transactions on Industry Applications, vol. 29, no. 6, 1993, pp. $1042-1046$.

[16] V. F. Fusco and P. A. Linden, "A Markov Chain Approach for Static Field Analysis," Microwave and Optical Technology Letters, vol. 1, 1988, pp. 216-220.

[17] A. E. Shadare, M. N. O. Sadiku and S. M. Musa, "Solution to Poisson's Equation in Rectangular Inhomogeneous Dielectric Media with the Markov Chain Monte Carlo," International Conference on Scientific Computing, 2017, pp. 10-15.

[18] K. Gu and M. N. O. Sadiku, "Absorbing Markov Chain Solution for Poisson's Equation," Proceedings of the IEEE Southeastcon, 2000, pp. 297-300.

[19]R. C. Garcia, M. N. O. Sadiku and K. Gu, "Applying Absorbing Markov Chains to Solve Poisson's Equation in Inhomogeneous Regions," Proceedings of the IEEE Southeastcon, 2001, pp. 166-168.

[20] A. E. Shadare, M. N. O. Sadiku and S. M. Musa, "Analysis of Microstrip Line using Markov Chain Monte Carlo," International Conference on Scientific Computing, 2015, pp. 135-139.

[21] M. N. O. Sadiku and R. C. Garcia, "Whole Field Computation Using Monte Carlo Method," International Journal of Numerical Modelling: Electronic Networks, Devices and Fields, vol. 10, 1997, pp. 303-312.

[22]M. N. O. Sadiku, Monte Carlo Methods for Electromagnetics. CRC Press, Boca Raton, FI, 2009.

[23]K. Asano, "Electrostatic potential and field in a cylindrical tank containing charged liquid," Proc. Inst. Elec. Eng., vol. 124, no. 12, December 1977, pp.12771281.

[24]M. N. O. Sadiku. "Monte Carlo solution of axisymmetric potential problems," Proc. of IEEE Industry Applications Society Annual Meeting, 1990. pp. 1894-1900. 